\title{
Ectonucleoside triphosphate diphosphohydrolase 6 expression in testis and testicular cancer and its implication in cisplatin resistance
}

\author{
YASUHIRO TADA $^{1}$, AKIRA YOKOMIZO $^{1}$, MASAKI SHIOTA $^{1}$, YOOHYUN SONG $^{1,2}$, \\ EIJI KASHIWAGI ${ }^{1}$, KENTARO KUROIWA $^{1}$, YOSHINAO ODA ${ }^{2}$ and SEIJI NAITO ${ }^{1}$
}

Departments of ${ }^{1}$ Urology and ${ }^{2}$ Anatomic Pathology, Graduate School of Medical Sciences, Kyushu University, Fukuoka, Japan

Received February 8, 2011; Accepted March 21, 2011

DOI: 10.3892/or.2011.1274

\begin{abstract}
The development of resistance to cisplatin during treatment of testicular cancer constitutes a major obstacle to the cure of testicular cancer. To resolve its mechanism will provide useful information for making clinical decisions. We found 4 and 15 gene expressions were, respectively, up-regulated and down-regulated in cisplatin-resistant testicular cancer NEC-8/DDP cells compared with their parental NEC- 8 cells (about 2.5 -fold) using cDNA microarray analysis. After screening, we selected the ENTPD6 among these 19 genes. ENTPD6 was less expressed in cancerous region compared with that in non-cancerous lesion. In addition, ENTPD6 expression in seminoma was higher than that in other testicular tumors. ENTPD6 expression was involved in cellular sensitivity to cisplatin through an interaction with E-cadherin. ENTPD6 is a promising molecular biomarker of cisplatin resistance in testicular cancer, and also a novel therapeutical target modulating cisplatin resistance in testicular cancer.
\end{abstract}

\section{Introduction}

Although testicular cancer is a relatively rare tumor, it is the most common cancer in young males (1). Cisplatin-based combination chemotherapy such as bleomycin, etoposide and cisplatin (BEP) plays an indispensable role in the treatment of testicular cancer $(2,3)$. Concurrently, about $80 \%$ of metastatic testicular cancer patients can be cured by chemotherapy and appropriate surgery. However, for those not cured, a method to overcome resistance to anticancer agents is required.

Correspondence to: Dr Seiji Naito, Department of Urology, Graduate School of Medical Sciences, Kyushu University, 3-1-1, Maidashi, Higashi-ku, Fukuoka 812-8582, Japan

E-mail: naito@uro.med.kyushu-u.ac.jp

Key words: cisplatin, E-cadherin, ectonucleoside triphosphate diphosphohydrolase 6 , testicular cancer, testis
Ectonucleoside triphosphate diphosphohydrolase (ENTPD) is a family of enzymes that catalyze the hydrolysis of phosphate residues of nucleotides (4). ENTPD family constitutes eight members that localize in the plasma membrane and/or in intracellular organelles with one or two transmembrane domains, and are secreted to extracellular space $(5,6)$. ENTPD family regulates nucleotide-mediated signaling by controlling the rate, amount and timing of nucleotide (e.g. ATP) degradation and ultimately, the nucleoside (e.g. adenosine) formation. Extracellular nucleotides/ nucleosides are shown to play a role in several physiological functions including haemostasis vascular and cardiac function (7-9). ENTPD6, a member of ENTPD family shows an intracellular localization such as the endoplasmic reticulum and Golgi apparatus, and the plasma membrane localization and could be secreted by proteolytic cleavage (10-12). However, its characteristics and roles in testis and testicular cancer remain unknown.

In this study, we aimed to identify the diagnostic and therapeutical molecular target of cisplatin resistance in testicular cancer. Then, we performed cDNA microarray analysis using human testicular cancer NEC-8 cells and the corresponding cisplatin-resistant NEC-8/DDP cells, and selected ENTPD6 as a candidate gene among several genes listed up by cDNA microarray analysis by semi-quantitative RT-PCR and quantitative real-time PCR. Furthermore, we characterized ENTPD6 expression in testis and testicular cancer, and elucidated the mechanism of involvement of ENTPD6 to cisplatin sensitivity.

\section{Materials and methods}

Cell culture. Human testicular cancer NEC-8 cells were cultured with RPMI-1640 and human bladder cancer T24 and KK47 cells were cultured with MEM (13). These media were purchased from Invitrogen (San Diego, CA, USA) and supplemented with $10 \%$ fetal bovine serum. Cisplatinresistant T24/DDP10 and KK47/DDP20 cells were established and maintained as described previously $(14,15)$. Cisplatinresistant derivatives of NEC-8 cells (NEC-8/DDP cells) were established by long-term culture under medium containing gradually increasing concentrations of cisplatin and main- 
tained under medium containing $4 \mu \mathrm{g} / \mathrm{ml}$ of cisplatin. The cell lines were maintained in a $5 \% \mathrm{CO}_{2}$ atmosphere at $37^{\circ} \mathrm{C}$.

Antibodies and plasmid. Antibodies against ENTPD6 (M03, clone 2D10) and E-cadherin were purchased from Abnova (Taipei, Taiwan) and BD Bioscinces (San Jose, CA, USA), respectively. Anti- $\beta$-actin antibody was purchased from Sigma (St. Louis, MO, USA). The ENTPD6 expression plasmid (pCMV6-AC-ENTPD6) expressing ENTPD6 protein was purchased from OriGene (Rockville, MD, USA). The shENTPD6 plasmids expressing shRNA against ENTPD6 (shENTPD6 \#1, pGFP-V-RS-shENTPD6 GI352789 and shENTPD6 \#2, pGFP-V-RS-shENTPD6 GI352791) were purchased from OriGene.

Cytotoxicity analysis. Cytotoxicity analysis was performed as described previously (17). NEC-8 cells $\left(2 \times 10^{3}\right)$ transfected with pCMV6-AC or pCMV-AC-ENTPD6 plasmid were seeded into 96-well plates. The following day, various concentration of cisplatin was added. After $48 \mathrm{~h}$, the surviving cells were stained with the alamarBlue assay (TREK Diagnostic Systems, Cleveland, $\mathrm{OH}, \mathrm{USA}$ ) for $180 \mathrm{~min}$ at $37^{\circ} \mathrm{C}$. The absorbances of the wells were measured using a plate reader (ARVO $^{\text {TM }}$ MX; Perkin Elmer Inc., Waltham, MA, USA).

cDNA microarray analysis. The procedure of cDNA microarray analysis was performed as described previously (18). Briefly, cDNA generation, hybridization, and data collection for cDNA microarray analyses were performed by the Incyte Corporation (Palo Alto, CA, USA). In brief, alterations in gene expression were evaluated by the reverse transcription of poly-(A) RNAs in the presence of Cy3 or Cy5 fluorescentlabeling dyes followed by hybridization to a UniGEM V 2.0 microarray chip. Each chip displays a total of 9182 elements, of which 8556 are unique gene/EST clusters. These unique gene/EST clusters can be further defined as 8412 annotated and 144 unannotated sequences. A 16-color log scale was used for visual representation and the differential expression $(\log 2)$ was determined for Cy3 signal: the signal of parental cell lines NEC-8 cells vs. Cy5 signal: the signal of resistant cell lines NEC-8/DDP cells as viewed in the Incyte GEM ${ }^{\mathrm{TM}}$ Tools software (Incyte Corporation). The subsets of genes were selected for further study based on differential Cy3/Cy5 expression ratios that were $\geq 3$.

Tissues and clinical data. Thirty-one patients with testicular cancer who provided sufficient tissue for the gene expression analysis were included in the study. Written informed consent was obtained from all subjects. The study was approved by the Institutional Review Board of Kyushu University. Immunohistochemical analysis was performed using samples from patients with or without testicular cancer. They underwent orchiectomy at Kyushu University Hospital, Japan. All testicular cancer specimens were completely reviewed to establish histological classification.

$R N A$ isolation and RT-PCR. Total RNA was prepared from cultured cells using an RNeasy Mini Kit (Qiagen, Hilden, Germany). First-strand cDNA was synthesized from $1.0 \mu \mathrm{g}$ of total RNA using a Transcriptor First Strand cDNA Synthesis
Kit (Roche Applied Science, Indianapolis, IN, USA) according to the manufacturer's protocol.

Semi-quantitative RT-PCR analysis. Semi-quantitative RT-PCR was performed as described previously (19). The ENTPD6 forward primer was 5'-ACTCCCACGTTAACCCACGA-3' and the reverse primer were 5'-AAACGCCTTCATCTGTTCC-3'. The reaction conditions had an initial denaturation step for $10 \mathrm{~min}$ at $95^{\circ} \mathrm{C}, 35 \mathrm{cycles}$ of $95^{\circ} \mathrm{C}$ x $30 \mathrm{sec}, 57^{\circ} \mathrm{C}$ x $30 \mathrm{sec}, 72^{\circ} \mathrm{C}$ x $30 \mathrm{sec}$, and a final extension step at $72^{\circ} \mathrm{C} \mathrm{x} 10 \mathrm{~min}$. The PCR products were separated by electrophoresis on $2 \%$ agarose gels, which were then stained with ethidium bromide. GAPDH was used as a control for RT-PCR. The GAPDH forward primer was 5'-ACCCAGAAGACTGTGGATGG-3' and the reverse primer was 5'-AGGGGTCTACATGGCAACTG-3'.

Quantitative real-time PCR. Quantitative real-time PCR was performed as described previously $(17,20)$. The synthesized cDNA was diluted 1:2 and $2.0 \mu 1$ of the diluted sample was used. Quantitative real-time PCR with TaqMan Gene Expression Assay (Applied Biosystems, Foster City, CA, USA) and TaqMan Gene Expression Master Mix (Applied Biosystems) was performed using an ABI 7900HT System. The expression level of each ENTPD6 gene was corrected by the corresponding GAPDH expression level. The results are representative of at least three independent experiments.

Western blot analysis. Whole-cell extracts were prepared as described previously $(17,20)$. The protein concentrations were quantified using a Protein Assay Kit (Bio-Rad, Hercules, CA, USA). Whole-cell extracts $(30 \mu \mathrm{g})$ were separated by SDS-PAGE and transferred to polyvinylidene difluoride microporous membranes (GE Healthcare Bio-Science, Piscataway, NJ, USA) using a semi-dry blotter. The blotted membranes were sequentially incubated with appropriate primary antibodies for $1 \mathrm{~h}$, and peroxidase-conjugated secondary antibodies for $40 \mathrm{~min}$ at room temperature. The bound antibodies were visualized using an ECL Kit (GE Healthcare Bio-Science) and the membranes were exposed to X-OMAT film (Kodak, Tokyo, Japan).

Immunohistochemistry. Immunohistochemical analysis was performed as described previously (21). The primary antibodies used in this study were anti-ENTPD6 antibody (mouse monoclonal, $3 \mu \mathrm{g} / \mathrm{ml}$ of concentration). Antigen retrieval was performed by microwave heating in citrate buffer (pH 6.0) for $20 \mathrm{~min}$.

Transfection. Transfection was performed as described previously $(17,20)$. Briefly, NEC-8 cells were transfected with DNA plasmid using Lipofectamine 2000 (Invitrogen), according to the manufacturer's protocol.

Co-immunoprecipitation assay. The co-immunoprecipitation assays were performed as described previously (22). Briefly, NEC-8 cells were lysed with buffer X, centrifuged at $21,000 \mathrm{~g}$ for $10 \mathrm{~min}$ at $4^{\circ} \mathrm{C}$ and the supernatants $(500 \mu \mathrm{g})$ were incubated overnight at $4^{\circ} \mathrm{C}$ with $2.0 \mu \mathrm{g}$ of anti-mouse IgG or anti-E-cadherin antibody. Then, immune-complex was incubated for $2 \mathrm{~h}$ at $4^{\circ} \mathrm{C}$ with $20 \mu \mathrm{l}$ of protein $\mathrm{A} / \mathrm{G}$ agarose 
Table I. The results of cDNA microarray analysis (NEC-8 vs. NEC-8/DDP).

\begin{tabular}{|c|c|c|c|}
\hline Gene name & Accession no. & Locus & Differential expression \\
\hline \multicolumn{4}{|l|}{ Up-regulated genes in NEC-8/DDP cells } \\
\hline Lymphocyte cytosolic protein 1 (L-plastin) & BF035921 & $13 q 14.3$ & -4.1 \\
\hline High density lipoprotein binding protein (vigilin) & NM_005336 & $2 q 37$ & -3.7 \\
\hline Spermidine/spermine N1-acetyltransferase & BF573292 & $\mathrm{Xp} 22.1$ & -3.6 \\
\hline Peptidylprolyl isomerase A (cyclophilin A) & AV713597 & 7p13-p11.2 & -3.0 \\
\hline \multicolumn{4}{|l|}{ Down-regulated genes in NEC-8/DDP cells } \\
\hline Cathepsin F & BE502253 & $11 \mathrm{q} 13$ & 9.6 \\
\hline Calbindin $1,(28 \mathrm{kD})$ & NM_004929 & $8 \mathrm{q} 21.3-\mathrm{q} 22.1$ & 4.8 \\
\hline $\begin{array}{l}\text { Hepatocellular carcinoma associated protein; breast cancer } \\
\text { associated gene } 1 \text { EST }\end{array}$ & BF969633 & Xp11.4-p11.1 & 4.6 \\
\hline TASP for testis-specific adriamycin sensitivity protein & BE219611 & $7 q 31.1-7 q 31.33$ & 4.6 \\
\hline $\begin{array}{l}\text { Homo sapiens mRNA; cDNA DKFZp434P086 } \\
\text { (from clone DKFZp434P086); partial c }\end{array}$ & AA813471 & & 4.4 \\
\hline Immediate early response 3 & N32077 & $6 \mathrm{p} 21.3$ & 3.6 \\
\hline ESTs & AW973290 & & 3.6 \\
\hline Protein phosphatase 1 , regulatory (inhibitor) subunit $1 \mathrm{~A}$ & XM_006813 & $12 q 13.2$ & 3.6 \\
\hline Mal, T-cell differentiation protein & NM_022439 & $2 q 13$ & 3.5 \\
\hline $\begin{array}{l}\text { Sjogren syndrome antigen A1 ( } 52 \mathrm{kD} \text {, ribonucleoprotein } \\
\text { autoantigen } \mathrm{SS}-\mathrm{A} / \mathrm{Ro})\end{array}$ & BE897247 & $11 \mathrm{p} 15.5$ & 3.4 \\
\hline Interferon induced transmembrane protein $3(1-8 \mathrm{U})$ & BF699055 & $11 \mathrm{p} 15.5$ & 3.2 \\
\hline RAB4, member RAS oncogene family & ВC004309 & $1 q 42-q 43$ & 3.2 \\
\hline $\begin{array}{l}\text { Ectonucleoside triphosphate diphosphohydrolase } 6 \\
\text { (putative function) }\end{array}$ & XM_009435 & $20 \mathrm{q} 11.2$ & 3.1 \\
\hline Heat shock $70 \mathrm{kD}$ protein $1 \mathrm{~B}$ & AF134726 & $6 \mathrm{p} 21.3$ & 3.0 \\
\hline Dihydropyrimidinase-like 4 & XM_011864 & $10 q 26$ & 3.0 \\
\hline
\end{tabular}

(Santa Cruz Biotechnology). The immunoprecipitated samples were washed three times with buffer $\mathrm{X}$ and the pre-immunoprecipitated samples $(50 \mu \mathrm{g})$ were subjected to Western blot analysis with the indicated antibodies.

\section{Results}

Nineteen genes were identified as differential-expressed genes between NEC-8 and NEC-8/DDP cells by cDNA microarray analysis. We established cisplatin-resistant NEC-8/DDP cells from testicular cancer NEC- 8 cells by long-term culture under medium containing gradually increasing concentrations of cisplatin. Then, NEC-8/DDP cells were about 2.5-fold resistant to cisplatin compared with NEC-8 cells (Fig. 1A). To reveal the characteristics of gene expression in NEC-8 and NEC-8/DDP cells, we performed cDNA microarray analysis using NEC- 8 and NEC-8/DDP cells. Then, microarray analysis revealed that 4 and 15 gene expressions were up-regulated and downregulated in NEC-8/DDP cells compared with NEC-8 cells, respectively, when 3 -fold change was employed as threshold value.

ENTPD6 expression is down-regulated in NEC-8/DDP cells compared with that in NEC-8 cells. Based on the results of microarray analysis, we validated the expression of differentially-expressed genes between NEC-8 and NEC-8/DDP cells (Table I). First, we screened several candidate genes (cathepsin $F$, calbindin 1, TASP for testis-specific adriamycin sensitivity protein, high density lipoprotein binding protein, spermidine/spermine N1-acetyltransferase, protein phosphatase 1 and ENTPD6) from the results of microarray analysis using semi-quantitative RT-PCR analysis. The results showed that the expression of any genes except ENTPD6 was similar between NEC-8 and NEC-8/DDP cells (data not shown). However, only ENTPD6 gene expression remarkably decreased in NEC-8/DDP cells as shown in Fig. 1B. Furthermore, this finding that ENTPD6 gene expression was reduced in cisplatin-resistant cells was confirmed by quantitative real-time PCR analysis. As shown in Fig. 1C, ENTPD6 gene expression was remarkably reduced in NEC-8/DDP as well as other cisplatin-resistant bladder cancer cells (T24/DDP10 and KK47/DDP20), which were established as described previously $(14,15)$. Finally, whether this difference of gene expression contributed to protein expression was confirmed by Western blotting. Three cisplatin-resistant cancer cells including NEC-8/ DDP, T24/DDP10 and KK47/DDP20 cells expressed less ENTPD6 protein than their parental cells (Fig. 1D). 
A

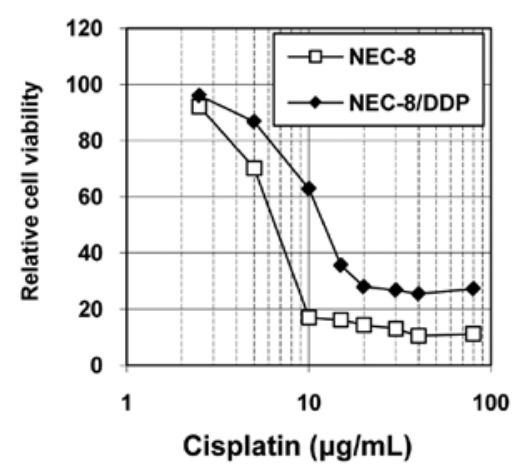

C

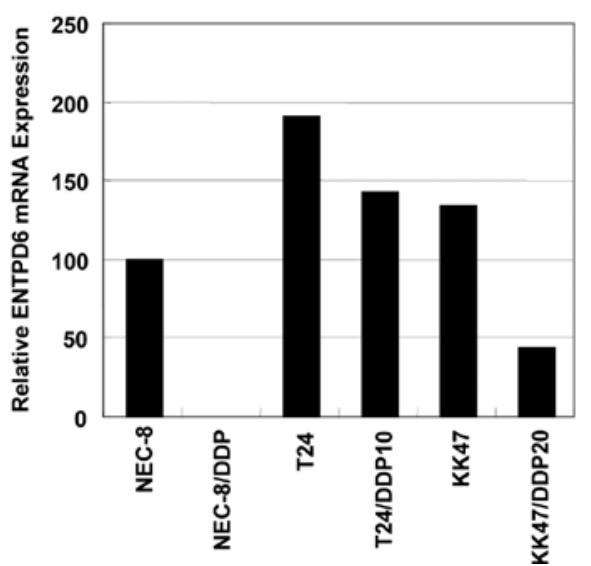

B

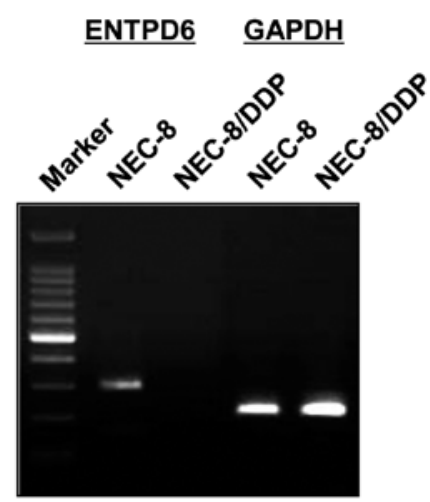

D

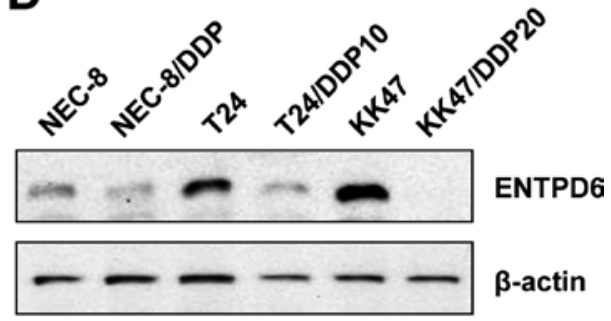

Figure 1. ENTPD6 is down-regulated in NEC-8/DDP cells compared with that in NEC-8 cells. (A) NEC-8 and NEC-8/DDP cells were seeded into 96-well plates. The following day, various concentrations of cisplatin were applied. After incubation for $48 \mathrm{~h}$, cell survival was analyzed by a cytotoxicity assay. Cell survival in the absence of cisplatin corresponds to 1 . All values are representative of at least three independent experiments. (B) Semi-quantitative RT-PCR was performed using cDNA from NEC-8 and NEC-8/DDP cells as template and the primers for ENTPD6 and GAPDH. (C) Quantitative real-time PCR was performed using cDNA from NEC-8, T24, KK47 and their cisplatin-resistant cells and the primers and probes for ENTPD6 and GAPDH. The ENTPD6 transcript levels were corrected by the corresponding GAPDH transcript levels. All values are representative of at least three independent experiments. Each transcript level from NEC-8 cells was set as 100. (D) Whole-cell extracts of NEC-8, T24, KK47 and their cisplatin-resistant cells were analyzed for ENTPD6 and $\beta$-actin (loading control) by SDS-PAGE and Western blotting with specific antibodies.

ENTPD6 is differentially expressed in testis and testicular cancer. To explore the relevance of ENTPD6 expression in testis and its malignant tumor, we compared ENTPD6 mRNA expression level between non-cancerous region and cancerous region using various testicular cancer tissues surgically obtained. First, we performed RT-PCR analysis. ENTPD6 mRNA expression in non-cancerous region and in cancerous region was detected in 9/9 (100\%) and 26/31 (83.9\%), respectively (data not shown). In addition, higher ENTPD6 mRNA expression in non-cancerous region compared with that in cancerous region was confirmed by quantitative real-time PCR (Fig. 2A). Subsequently, we performed immunohistochemistry against human normal testis and testicular cancer. ENTPD6 localized mainly in cytosol and plasma membrane and was expressed to a weak or moderate extent in spermatoblast, seminiferous tubule, seminoma and its metastatic site, but abundantly expressed in Leydig cells in stroma (Fig. 2B).

ENTPD6 is differentially expressed between seminoma and other testicular tumors. To investigate ENTPD6 expression in testicular cancer, we analyzed ENTPD6 mRNA expression in testicular cancer. ENTPD6 mRNA expressions in cancerous region of various testicular cancer tissues were measured. As shown in Fig. 3, ENTPD6 expression was affected by tissuetype of testicular cancer (seminoma vs. other testicular tumor).

ENTPD6 is involved in cisplatin resistance through an interaction with E-cadherin. Finally, we investigated a mechanism of the relationship between ENTPD6 expression and cisplatin resistance. When ENTPD6 was overexpressed in NEC-8 cells by transfecting with ENTPD6 expression plasmid, NEC-8 cells were more sensitive to cisplatin (Fig. 4A). Secreted-form and membrane-bound form of ENTPD6 are known (12). E-cadherin is also known to localize in plasma membrane and to be involved in cisplatin resistance $(23,24)$. Then, we investigated the possibility of interaction between E-cadherin and ENTPD6. When E-cadherin was immunoprecipitated using anti-E-cadherin antibody, ENTPD6 was detected in the resulting-immunoprecipitated complex (Fig. 4B). Furthermore, when ENTPD6 expression was suppressed using two kinds of ENTPD6 specific-shRNA, E-cadherin expression was reduced (Fig. 4C), suggesting that ENTPD6 is implicated in 
A

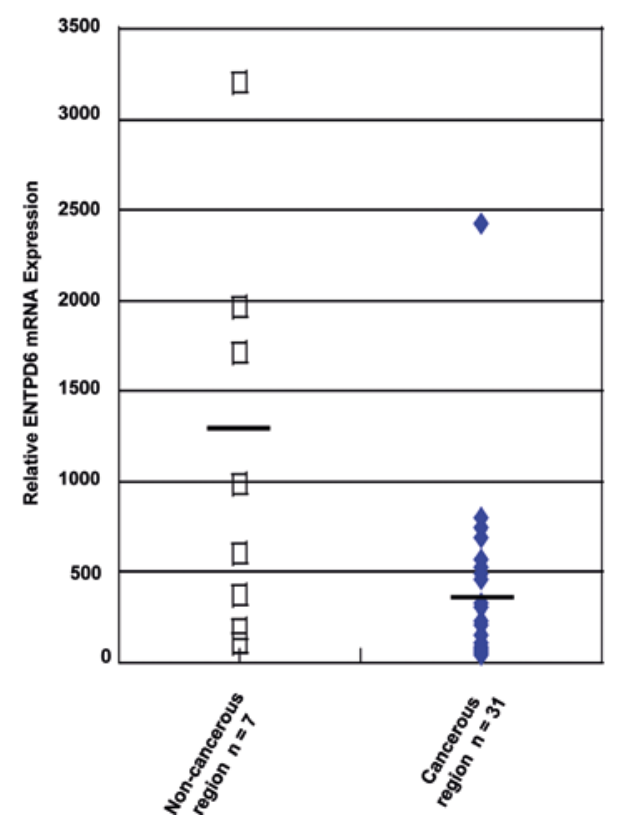

C

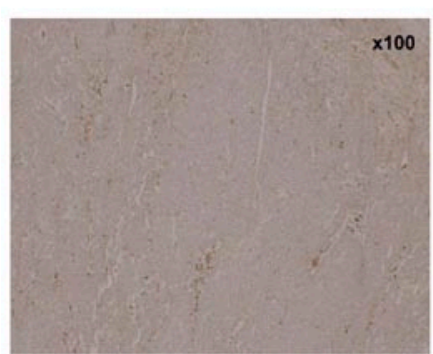

Seminoma

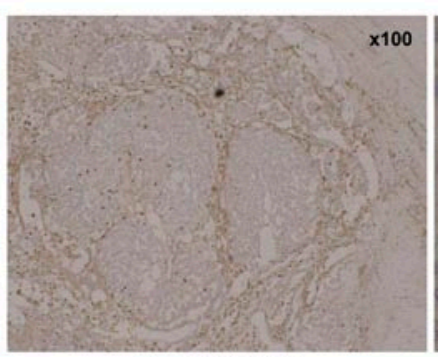

Metastatic site

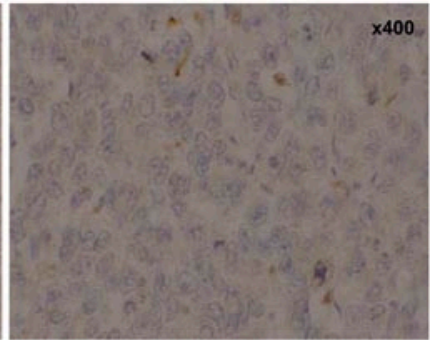

Seminoma

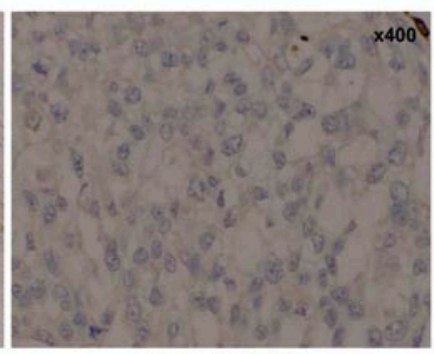

Metastatic site
E-cadherin expression probably through E-cadherin protein stability.

\section{Discussion}

This study revealed that ENTPD6 is a candidate of key molecule determining cisplatin resistance in testicular cancer. Although ENTPD6 is so far reported to be expressed in various tissues including muscle, heart and in fetal brain (25), ENTPD6 expressions in testis and testicular cancer are unknown. In this study, ENTPD6 was detected in all testicular tissues of non-cancerous region and in most cancerous region, indicating that ENTPD6 plays a role in testis and testicular cancer. ENTPD6 is known to catalyze ATP and ADP to AMP. Since extracellular ATP is supposed to regulate many cellular
B

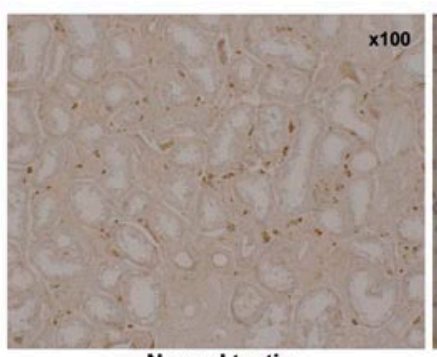

Normal testis

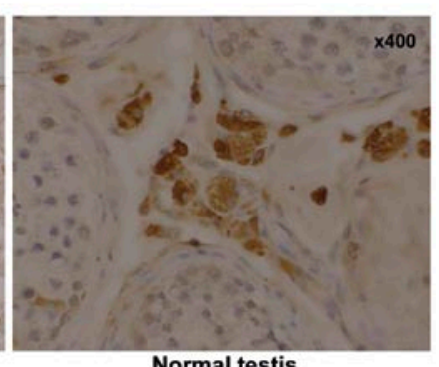

Normal testis

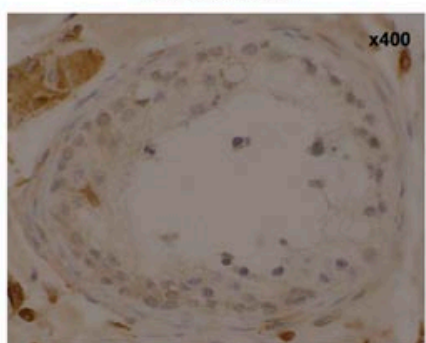

Normal seminiferous tubule

Figure 2. ENTPD6 is differentially expressed in testis and testicular cancer. (A) Quantitative real-time PCR using was performed cDNA from various non-cancerous region and cancerous region and the primers and probes for ENTPD6 and GAPDH. The ENTPD6 transcript levels were corrected by the corresponding GAPDH transcript levels. All values are representative of at least three independent experiments. The transcript level of NEC-8 cells was set as 100. (B and C) Immunohistochemistry against ENTPD6 in testis (B) and seminoma $(\mathrm{C})$ is shown.

functions in various tissues, ENTPD6 in testis may play an indispensable role in its function.

Cisplatin resistance is thought to be complex and multicausal phenomenon. Many resistance-related molecules including detoxifying enzymes, drug-efflux pumps, DNA repair enzymes and apoptosis-related genes have been reported $(26,27)$. In this study, we identified that ENTPD6 may be a factor determining cisplatin sensitivity in testicular cancer. Seminoma is known to be highly-sensitive to chemotherapy including cisplatin-based one. This study revealed that ENTPD6 expression in seminoma was higher than that in other testicular tumor, suggesting that high sensitivity to cisplatin in seminoma might derive from relative-high expression of ENTPD6. Recently, epithelial-mesenchymal transition (EMT) is focused on in relationship with the resistance to 


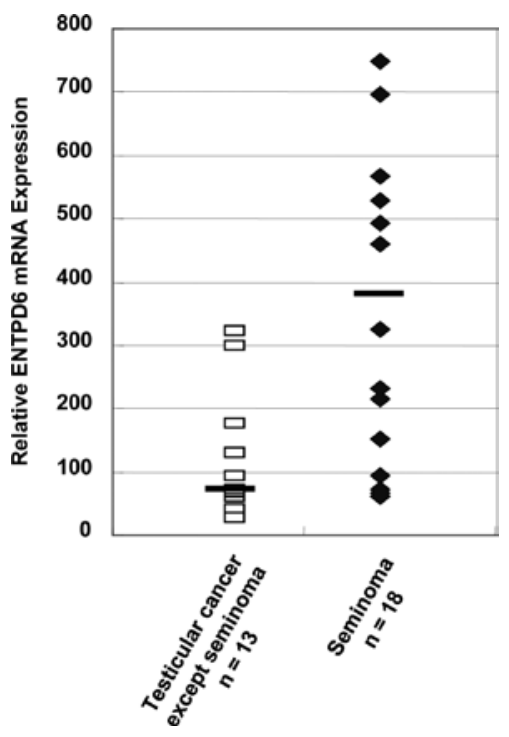

Figure 3. ENTPD6 is differentially expressed between non-seminoma and seminoma. Quantitative real-time PCR using was performed cDNA from various non-seminoma and seminoma tissues and the primers and probes for ENTPD6 and GAPDH. The ENTPD6 transcript levels were corrected by the corresponding GAPDH transcript levels. All values are representative of at least three independent experiments. The transcript level of NEC-8 cells was set as 100 . anticancer therapeutics. E-cadherin is a key player of EMT, which expression is known to be regulated by Twist1 and other transcription factors including Snail and Slug. We and others previously found that Twist1 is a key player in cisplatin resistance (28-30), suggesting that Twistl may exert its cisplatin-resistant function through a regulation of gene expressions of E-cadherin and others. E-cadherin recently is reported to be closely implicated in and inversely correlated with cisplatin resistance $(23,24)$. In this study, ENTPD6 was found to interact with E-cadherin and to regulate E-cadherin expression. Although its mechanism remained unclear, it was suspected to regulate E-cadherin expression through protein stability because ENTPD6 was shown to interact with E-cadherin. Therefore, ENTPD6 may exert cisplatin resistance through a regulation of E-cadherin expression.

E-cadherin is known to be involved in carcinogenesis and tumor progression in various cancers (31). In mouse model of $\beta$-cell tumors of the pancreas, E-cadherin loss is a causal prerequisite for progression from adenoma to invasive carcinomas (32). In addition to many reports about E-cadherin expression in cancer by others, we have reported E-cadherin expression in prostate cancer $(21)$ and renal cancer $(33,34)$ and the methylation of E-cadherin promoter in bladder cancer (35). Then, the relationship between ENTPD6 and E-cadherin
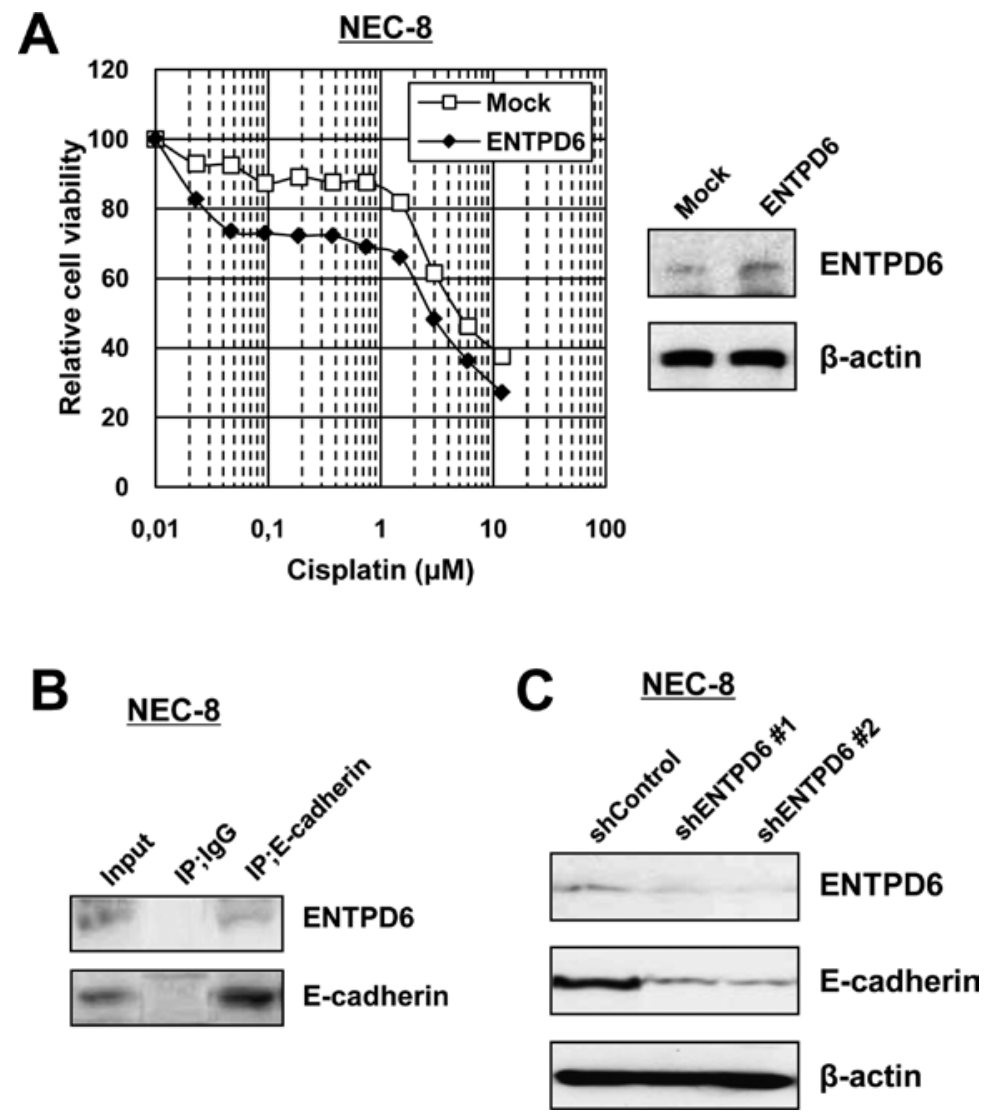

Figure 4. ENTPD6 is involved in cisplatin resistance through an interaction with E-cadherin. (A) NEC-8 cells were transfected with Mock or ENTPD6 expression plasmid. Various concentration of cisplatin was applied at $24 \mathrm{~h}$ after transfection, and then the cell viability was measured at $48 \mathrm{~h}$ after cisplatin addition. Values represent means from three independent experiments. NEC-8 cells were transiently transfected with Mock or ENTPD6 expression plasmid, and then the cells were harvested at $48 \mathrm{~h}$ after transfection. ENTPD6 and $\beta$-actin (loading control) expression was detected by Western blot analysis using anti-ENTPD6 and anti- $\beta$-actin antibodies. (B) Whole-cell extracts (500 $\mu \mathrm{g})$ were immunoprecipitated with $2.0 \mu \mathrm{g}$ of mouse IgG or anti-E-cadherin antibody. The pre-immunoprecipitants (input) and immunoprecipitants were subjected to SDS-PAGE, and Western blotting was performed using the indicated antibodies. (C) Whole-cell extracts of NEC-8 cells transfected with the indicated shRNA plasmid were analyzed for ENTPD6, E-cadherin and $\beta$-action (loading control) by SDS-PAGE and Western blotting with specific antibodies. 
might suggest its role of ENTPD6 in cancer carcinogenesis and progression.

In conclusion, we have revealed that ENTPD6 may be a tumor suppression gene and a determinant of cisplatin resistance in testicular cancer. Therefore, ENTPD6 is a promising diagnostic molecule of cisplatin resistance in testicular cancer and a molecular therapeutic target of testicular cancer.

\section{Acknowledgements}

This study was in part supported by Health Sciences Research Grants for Clinical Research for Evidenced Based Medicine and Grants-in-Aid for Cancer Research (016), from the Ministry of Health, Labor and Welfare, Japan; Kakenhi grants (22591769) from The Ministry of Education, Culture, Sports, Science, and Technology of Japan (MEXT), Japan; Research Promotion Grant from The Japanese Foundation for Prostate Research, Japan; Research Promotion Grant from The Tokyo Biochemical Research Foundation, Japan and Grant-in-Aid for Cancer Research from The Fukuoka Foundation for Sound Health, Japan. We would like to thank Ms. Noriko Hakoda, Ms. Hitomi Matoba, Ms. Miho Ushijima and Ms. Seiko Kamori for their technical assistances.

\section{References}

1. Garner MJ, Turner MC, Ghadirian $\mathrm{P}$ and Krewski D Epidemiology of testicular cancer: an overview. Int J Cancer 116: 331-339, 2005.

2. Williams SD, Birch R, Einhorn LH, et al: Treatment of disseminated germ-cell tumors with cisplatin, bleomycin, and either vinblastine or etoposide. N Engl J Med 316: 1435-1440, 1987.

3. Feldman DR, Bosl GJ, Sheinfeld J and Motzer RJ: Medical treatment of advanced testicular cancer. JAMA 299: 672-684, 2008.

4. Zimmermann H: Ectonucleotidases: some developments and a note on nomenclature. Drug Develop Res 52: 44-56, 2001.

5. Robson S, Sévigny J and Zimmermann H: The E-NTPDase family of ectonucleotidases: structure function relationships and pathophysiological significance. Purinergic Signal 2: 409-430, 2006.

6. Bigonnesse F, Levesque SA, Kukulski F, et al: Cloning and characterization of mouse nucleoside triphosphate diphosphohydrolase-8. Biochemistry 43: 5511-5519, 2004.

7. Burnstock G: Dual control of local blood flow by purines. Ann N Y Acad Sci 603: 31-44, 1990.

8. Kunapuli SP and Daniel JL: P2 receptor subtypes in the cardiovascular system. Biochem J 336: 513-523, 1998.

9. Ralevic V and Burnstock G: Involvement of purinergic signalling in cardiovascular diseases. Drug News Perspect 16: 133-140, 2003.

10. Wang TF and Guidotti G: Golgi localization and functional expression of human uridine diphosphatase. J Biol Chem 273: 11392-11399, 1998.

11. Biederbick A, Kosan C, Kunz J and Elsasserm H: First apyrase splice variants have different enzymatic properties. J Biol Chem 275: 19018-19024, 2000.

12. Mulero JJ, Yeung G, Nelken ST and Ford JE: CD39-L4 is a secreted human apyrases, specific for the hydrolysis of diphosphates. J Biol Chem 274: 20064-20067, 1999.

13. Yamamoto $\mathrm{T}$, Komatsubara S, Suzuki $\mathrm{T}$ and Oboshi $\mathrm{S}$ In vitro cultivation of human testicular embryonic carcinoma and establishment of new cell line. Gann 70: 677-680, 1979.

14. Kotoh S, Naito S, Yokomizo A, et al: Increased expression of DNA topoisomerase I gene and collateral sensitivity to camptothecin in human cisplatin-resistant bladder cancer cells. Cancer Res 54: 3248-3252, 1994.
15. Kotoh S, Naito S, Yokomizo A, Kohno K, Kuwano M and Kumazawa J: Enhanced expression of gamma-glutamylcysteine synthetase and glutathione $\mathrm{S}$-transferase genes in cisplatin-resistant bladder cancer cells with multidrug resistance phenotype. J Urol 157: 1054-1058, 1997.

16. Shiota M, Yokomizo A, Tada Y, et al: P300/CBP-associated factor regulates Y-box binding protein-1 expression and promotes cancer cell growth, cancer invasion and drug resistance. Cancer Sci 101: 1797-1806, 2010.

17. Shiota M, Yokomizo A, Kashiwagi E, et al: Foxo3a expression and acetylation regulate cancer cell growth and sensitivity to cisplatin. Cancer Sci 101: 1177-1185, 2010.

18. Tsunoda T, Koga H, Yokomizo A, et al: Inositol 1,4,5-trisphosphate (IP3) receptor type1 (IP3R1) modulates the acquisition of cisplatin resistance in bladder cancer cell lines. Oncogene 24: 1396-1402, 2005.

19. Tada Y, Wada M, Kuroiwa K, et al: MDR1 gene overexpression and altered degree of methylation at the promoter region in bladder cancer during chemotherapeutic treatment. Clin Cancer Res 6: 4618-4627, 2000.

20. Shiota M, Yokomizo A, Tada Y, et al: Castration resistance of prostate cancer cells caused by castration-induced oxidative stress through Twist1 and androgen receptor overexpression. Oncogene 29: 237-250, 2010.

21. Song Y, Oda Y, Hori M, et al: N-myc downstream regulated gene-1/Cap43 may play an important role in malignant progression of prostate cancer, in its close association with E-cadherin. Hum Pathol 41: 214-222, 2010.

22. Shiota M, Yokomizo A, Tada Y, et al: Peroxisome proliferatoractivated receptor gamma coactivator-1alpha interacts with the androgen receptor (AR) and promotes prostate cancer cell growth by activating the AR. Mol Endocrinol 24: 114-127, 2010.

23. Koppikar P, Lui VW, Man D, et al: Constitutive activation of signal transducer and activator of transcription 5 contributes to tumor growth, epithelial-mesenchymal transition, and resistance to epidermal growth factor receptor targeting. Clin Cancer Res 14: 7682-7690, 2008.

24. Arumugam T, Ramachandran V, Fournier KF, et al: Epithelial to mesenchymal transition contributes to drug resistance in pancreatic cancer. Cancer Res 69: 5820-5828, 2009.

25. Braun N, Fengler S, Ebeling C, Servos J and Zimmermann H: Sequencing, functional expression and characterization of rat NTPDase6, a nucleoside diphosphatase and novel member of the ecto-nucleoside triphosphate diphosphohydrolase family. Biochem J 351: 639-647, 2000.

26. Naito S, Yokomizo A and Koga $\mathrm{H}$ : Mechanisms of drug resistance in chemotherapy for urogenital carcinoma. Int J Urol 6: 427-439, 1999.

27. Naito S, Koga H, Yokomizo A, et al: Molecular analysis of mechanisms regulating drug sensitivity and the development of new chemotherapy strategies for genitourinary carcinomas. World J Surg 24: 1183-1186, 2000.

28. Shiota M, Izumi H, Onitsuka T, et al: Twist promotes tumor cell growth through YB-1 expression. Cancer Res 68: 98-105, 2008.

29. Shiota M, Izumi H, Tanimoto A, et al: Programmed cell death protein 4 down-regulates Y-box binding protein-1 expression via a direct interaction with Twist1 to suppress cancer cell growth. Cancer Res 69: 3148-3156, 2009.

30. Zhou WL, Wang Y, Zhou XL, Zhang YS and Chen ZT: Short interfering RNA directed against TWIST, a novel zinc finger transcription factor, increases A549 cell sensitivity to cisplatin via MAPK/mitochondrial pathway. Biochem Biophys Res Commun 369: 1098-1102, 2008.

31. Schmalhofer O, Brabletz S and Brabletz T: E-cadherin, betacatenin, and ZEB1 in malignant progression of cancer. Cancer Metastasis Rev 28: 151-166, 2009.

32. Perl AK, Wilgenbus P, Dahl U, Semb H and Christofori G: A causal role for E-cadherin in the transition from adenoma to carcinoma. Nature 392: 190-193, 1998.

33. Kuroiwa K, Konomoto T, Kumazawa J, Naito S and Tsuneyoshi M: Cell proliferative activity and expression of cell-cell adhesion factors (E-cadherin, alpha-, beta-, and gammacatenin, and p120) in sarcomatoid renal cell carcinoma. J Surg Oncol 77: 123-131, 2001.

34. Migita T, Oda Y, Masuda K, et al: Inverse relationship between E-cadherin and p27Kip1 expression in renal cell carcinoma. Int J Oncol 33: 41-47, 2008.

35. Tada Y, Wada M, Taguchi K, et al: The association of deathassociated protein kinase hypermethylation with early recurrence in superficial bladder cancers. Cancer Res 62: 4048-4053, 2002. 